\title{
U1-RNP and Toll-like receptors in the pathogenesis of mixed connective tissue disease Part II. Endosomal TLRs and their biological significance in the pathogenesis of mixed connective tissue disease
}

\author{
Agnieszka Paradowska-Gorycka \\ Department of Biochemistry and Molecular Biology, Institute of Rheumatology, Warsaw, Poland
}

\begin{abstract}
Mixed connective tissue disease (MCTD) is a chronic autoimmune immunopathological disease of unknown etiology, which is characterized by the presence of various clinical symptoms and the presence of autoantibodies against U1-RNP particles. The U1-RNP component engages immune cells and their receptors in a complex network of interactions that ultimately lead to autoimmunity, inflammation, and tissue injury. The anti-U1-RNP autoantibodies form an immune complex with self-RNA, present in MCTD serum, which can act as endosomal Toll-like receptor (TLR) ligands. Inhibition of TLRs by nucleic acids is a promising area of research for the development of novel therapeutic strategies against pathogenic infection, tumorigenesis and autoimmunity. In this review we summarize current knowledge of endogenous TLRs and discuss their biological significance in the pathogenesis of MCTD. In part I we described the structure, biological function and significance of the U1-RNP complex in MCTD.
\end{abstract}

Key words: pathogenesis, mixed connective tissue diseases (MCTD), TLR receptors.

\section{Introduction}

In 1972, Sharp et al. [1] proposed a new disease, termed mixed connective tissue diseases (MCTD), which is a rare chronic inflammatory systemic autoimmune syndrome, involving complex interactions between disease-related phenomena, including inflammation, proliferative vascular arteriopathy, thrombotic events, dyslipidemia and humoral autoimmune processes [2, 3]. There has been some controversy over whether MCTD is truly a distinct disease, as it shares some symptoms with systemic lupus erythematosus (SLE) and others.

Mixed connective tissue disease, like other autoimmune connective tissue diseases, is characterized by the stimulation of the immune system with the production of autoreactive autoantibodies against nuclear and cytoplasmic components, often macromolecular complexes of proteins and nucleic acids [4-6]. The two major groups of autoantibody populations in MCTD are targeted either against antinuclear antibodies (ANA) or against the U1 small nuclear ribonucleoprotein antigen (U1-snRNP, which is a complex of U1-RNA and small nuclear RNP). The conventional function for U1-RNP is in splicing, which is a key process for mRNA maturation, particularly in higher eukaryotes, where most protein-coding transcripts contain multiple introns. Originally, autoreactivity in patient sera against the U1-RNP complex was named anti-U1-RNP [7-9]. Although these autoantibodies are specific for the disease, their exact pathogenic role or otherwise is not fully understood. However, recent studies demonstrated that components of the U1-RNP complex may participate directly in provoking the antiU1-RNP responses. Moreover, anti-U1-RNP autoantibod-

Address for correspondence:

Agnieszka Paradowska-Gorycka, Department of Biochemistry and Molecular Biology, Institute of Rheumatology, Spartanska 1,

02-637 Warsaw, Poland, e-mail: paradowska_aga@interia.pl

Submitted: 12.03.2015; Accepted: 11.06.2015 
ies have been found to interact with lung tissue, thus significantly contributing to disease features $[8,10,11]$.

The clinical symptoms and the presence of autoantibodies suggested that many of the immunological factors may also be involved in MCTD. These factors include immune cell activation via innate signaling through Toll-like receptors (TLRS) and other innate immune receptors, modification of the RNP antigen and its associated RNAs, B cell hyperactivity, abnormal activation of T cells and defects in the clearance of apoptotic cells and immune complexes [12, 13]. MCTD-associated autoantigens are recognized by endosomal TLRs such as TLR3, 7, 8 and 9 , activation of which leads to downstream effects important in the generation of the disease phenotype. Moreover, the ability of U1-RNA to act as a TLR ligand may serve as an important link between innate immunity and the development of the anti-U1-RNP immune responses. Functionally, TLRs have been classified as essential components of the immune system that activate multiple inflammatory pathways and coordinate systemic defense against microbial pathogens. Engage- ment of TLRs with their ligands results in activation of several downstream intracellular pathways, leading to activation of innate and adaptive immune system cells. TLRs control induction of effective adaptive immune responses at several levels, including induction of T-cell priming and dendritic cell (DC) maturation. Despite their beneficial roles in detecting microbial/viral infections, inappropriate engagement of these receptors on B cells may initiate and/or perpetuate autoimmunity. Additionally, inhibition of activation of the endosomal TLRs and their signaling pathway or blocking of ligands binding to TLRs might reveal new therapeutics as anti-inflammatory agents, many of which are in the preclinical, first and third phase of application. Quinazoline is an antiinflammatory TLS agonist which inhibits TLRs 7/8/9 and inhibits disease progression of SLE and other autoimmune disease in animal models [14]. Furthermore, chloroquine, an old drug used in MCTD patients, inhibits endosomal TLR-dependent activation of immune cells and prevents the interaction of CPG with TLR9. Moreover, these anti-inflammatory agents may provide a safe and

Table I. Characteristics of the TLR family

\begin{tabular}{|c|c|c|c|c|}
\hline TLR & Localization & $\begin{array}{l}\text { Chromosomal } \\
\text { localization }\end{array}$ & Ligand & Cell types \\
\hline TLR1 & extracellular & $4 p 14$ & $\begin{array}{l}\text { Bacteria: peptidoglycan, } \\
\text { lipoproteins, LTA } \\
\text { Fungi: zymosan }\end{array}$ & DCs, B cells \\
\hline TLR2 & extracellular & $4 q 32$ & $\begin{array}{l}\text { Bacteria: peptidoglycan, lipoteichoic, } \\
\text { lipoproteins, lipoarabinomannan }\end{array}$ & $\begin{array}{l}\text { mDCs, monocytes, epithelia cells, } \\
\text { microglia cells }\end{array}$ \\
\hline TLR3 & intracellular & $4 q 35$ & Viruses: dsRNA & $\begin{array}{c}\text { mDCs, NK cells, astrocytes, neural } \\
\text { cells }\end{array}$ \\
\hline TLR4 & extracellular & $9 q 33.1$ & $\begin{array}{l}\text { Bacteria: LPS } \\
\text { Viruses: RSV fusion protein } \\
\text { Fungi: mannan } \\
\text { Protozoa: } \\
\text { glycoinositolphospholipids }\end{array}$ & $\begin{array}{l}\text { mDCs, macrophages, monocytes, } \\
\text { endothelial and epithelial cells }\end{array}$ \\
\hline TLR5 & extracellular & $1 q 41-q 42$ & Bacteria: flagellin & DCs, T cells, NK cells, epithelial cells \\
\hline TLR6 & extracellular & $4 p 14$ & Bacteria: lipoproteins & $\begin{array}{c}\text { B cells, NK cel, monocytes, epitelial } \\
\text { cells }\end{array}$ \\
\hline TLR7 & intracellular & Xp22.3 & Viruses: ssRNA & pDCs, B cells, monocytes \\
\hline TLR8 & intracellular & Xp22 & Viruses: ssRNA & T cells, NK cells, B cells, monocytes \\
\hline TLR9 & intracellular & $3 p 21.3$ & $\begin{array}{c}\text { Bacteria: CpG DNA } \\
\text { Viruses: CpG DNA } \\
\text { Protozoa: CpG DNA, haemozoin }\end{array}$ & $\begin{array}{c}\text { pDCs, B cells, NK cells, macrophages, } \\
\text { epitelial cells }\end{array}$ \\
\hline TLR10 & extracellular & $4 p 14$ & Unknown & pDCs, mDCs, B cells \\
\hline $\begin{array}{l}\text { TLR11 } \\
\text { (Mouse) }\end{array}$ & extracellular & $14 ; 14$ C1 & $\begin{array}{l}\text { Uropathogenic bacteria } \\
\text { Protozoa: profilin-like molecule }\end{array}$ & DCs, macrophages, epithelial cells \\
\hline $\begin{array}{l}\text { TLR12 } \\
\text { (Mouse) }\end{array}$ & extracellular & $4 ; 4$ D2.2 & Profilin & pDCs, macrophages \\
\hline $\begin{array}{l}\text { TLR13 } \\
\text { (Mouse) }\end{array}$ & intracellular & $X ; X D$ & Bacteria: RNA & $\begin{array}{l}\text { astrocytes, DCs, endothelial cells, } \\
\text { ependymal cells, macrophages }\end{array}$ \\
\hline
\end{tabular}


effective therapeutic approach for certain inflammatory and autoimmune diseases $[4,6,13,15]$.

\section{Toll-like receptors physiology}

Pattern recognition receptors (PRRs) are germlineencoded innate immune receptors that were originally reported as sensors for pathogen-associated molecular patterns (PAMPs).

Pattern recognition receptors can also recognize endogenous molecules released in response to stress or tissue damage, thus behaving as sensors of alarmins. Moreover, PRR engagement promotes the activation of innate and adaptive immune responses. Members of the TLR family are PRRs that recognize many pathogen-derived macromolecules, ranging from bacterial and yeast cell wall components to viral and bacterial nucleic acids $[6,13,16]$. The TLR family (Table I) is one of the largest and most well-studied in terms of known ligands, downstream signaling pathways, and functional relevance. Human TLRs are evolutionarily conserved homologs of the Drosophila melanogaster Toll protein, which is involved in embryonic development in D. melanogaster. At least ten functional TLRs (TLR1 to TLR10) have been identified in humans, whereas mice expressed twelve TLRs (TLR1 to TLR9 and TLR11 to TLR13). Ligands have been identified for all TLRs except for human TLR10, mouse TLR12 and mouse TLR13. The first described ligands for TLRs were of bacterial origin, such as peptidoglycan for TLR2 or lipopolysaccharides for TLR4, but since the discovery of double-stranded RNA (dsRNA) as the ligand for endosomal TLR3 it has been recognized that TLRs also have a role in the host defense against viruses. TLR1, TLR2, TLR4, TLR5, TLR6 and TLR11 reside at the plasma membrane, where they recognize molecular components located on the surface of pathogens. By contrast, TLR3, TLR7, TLR8 and TLR9, potent activators of DCs and B cells, are found intracellularly, where they mediate recognition of bacterial and viral nucleic acids released during intracellular infection. TLRS 7 and 8 are specific for single-stranded RNA (sSRNA), TLR9 for unmethylated $\mathrm{CpG}$ motifs within dsDNA, and the recently discovered TLR13 (an orphan receptor in mice) detects conserved 23s ribosomal RNA. While TLR3 is able to distinguish between pathogen and self-nucleic acids on the basis of distinct molecular structures, TLR7/8 and 9 do not have this feature [16]. The endosomal localization of the nucleic acid-sensing endosomal TLRs is believed to contribute to the distinction between endogenous nucleic acids and those of foreign origin [17-21]. Both extracellular and endosomal TLRs are homologous in the sequence of their ectodomain, a feature that is in sharp contrast with the diversity of the ligands they recognize.
Toll-like receptors are type I transmembrane glycoprotein receptors, with molecular weights ranging from 90 to $115 \mathrm{kDa}$, which on activation form homodimers or heterodimers to activate signaling. All TLR family members are composed of a ligand-binding ectodomain containing 18-25 tandem copies of leucine-rich repeats (LRRs), a transmembrane domain, and a conserved cytoplasmic toll/interleukin-1 receptor (TIR) domain. The signal transmembrane $\alpha$-helix portion and the TIR domain are homologous to those of the human interleukin-1 receptor (IL-1R) and human interleukin-18 receptor (IL-18R). The leucine-rich repeat (LLR) domain is important for ligand binding and associated signaling and is a common feature of PRRs.

Toll-like receptors display different amino acid composition within these modules, leading to structural conformation variations that allow for ligand interaction. Binding of ligands to the extracellular domains of TLRs causes rearrangement of the receptor complexes and triggers the recruitment of specific adaptor proteins to the intracellular TIR domains [22]. Toll-like receptor signaling involves five adaptor proteins such as myeloid differentiation factor 88 (MyD88), the TIR domain containing adaptor protein inducing interferon- $\alpha / \beta$ (TRIF; also known as TICAM1), MyD88-adaptor-like (MAL, also known as TIRAP), the TRIF-related adaptor molecule (TRAM; also known as TICAM2) and the sterile $\alpha$ - and armadillo-motif containing protein (SARM), which was recently identified as the fifth member of the TLR adaptor protein family and acts as a specific inhibitor of TRIF-dependent TLR signaling. Of these, MyD88 and TRIF function as signaling adaptors that mediate interaction to downstream kinases, whereas TRAM and TIRAP translocate MyD88 or TRIF to the activated TLRs. On the other hand, adaptor SARM functions as a negative regulator of TRIF signaling [23-26]. The MyD88 pathway is induced by all of the TLRs except TLR3 and TLR4 and leads to the activation of NF- $\kappa \mathrm{B}$ and ultimately production of cytokines such as TNF- $\alpha$ and IL 12p40 and upregulation of co-stimulatory molecules. The other pathway is activated only by TLR3 and TLR4 and is referred to as the MyD88-independent or TRIF-dependent pathway. TRIF signals to the interferon regulatory factor (IRF) family of transcription factors, leading to production of type I interferon and other pro-inflammatory cytokines. In addition to the pro-inflammatory signaling molecules downstream of the TLRs, there are also negative regulators of TLR signaling (such as A20 and IkBa) that operate at multiple levels to suppress inflammation [27, 28].

The high levels and broadest spectra of TLR expression have been observed in a variety of cell types in the immune system, including monocytes, macrophages, DCs, neutrophils, B- and T-cells, natural killer (NK) cells, 
mast cells and keratinocytes. Moreover, TLR expression has also been identified in synoviocytes, fibroblasts, osteoblasts, osteoclasts, chondrocytes and epithelial cells [29]. To identify more accurately potential sites of action, real-time quantitative PCR analyses of the expression of TLRs in human and mouse tissues have been performed. These studies have demonstrated that TLRs mainly express on all peripheral blood leukocytes, including CD4+ and CD8+ T cells and B cell populations and spleen tissue cells involved in immune function, but also on other tissues, such as ovaries, prostate, pancreas, placenta and testis [30, 31]. Epithelial cells also play additional key roles through TLR expression responding to pathogenic molecules. Thus, epithelial cells are more than just a barrier located at potential sites of entry, such as the skin, respiratory, intestinal and genitourinary tracts [32]. While the endosomal TLRs can be found inside cells expressing other non-endosomal TLRS, TLR7 and 9 are seldom found in the same type of cells as TLR3, but not on the same cell population. TLR7 and 9 are expressed on plasmacytoid DCs (pDCs) and bone marrow-derived DCs. In contrast, TLR3 is expressed on myeloid DCs (mDCs), specifically in human monocyte-derive DCs and CD11+ blood DCs as well as in murine CD8 $\alpha+$ DCs [17, 33]. Interestingly, TLR3 is also predominantly expressed in brain, heart, lung and muscle, suggesting that TLR3 may play a role in the anti-infection reaction in these tissues [34]. Furthermore, tissue expression of TLR7/8 and TLR9 is observed in the spinal cord, spleen and lung and the lymph nodes, tonsils and peripheral blood mononuclear cells, respectively [6].

\section{Biologic functions of Toll-like receptors}

Functionally, TLRs have been classified as essential components of the immune system that activate multiple inflammatory pathways and coordinate systemic defense against microbial pathogens. Engagement of TLRs with their ligands results in activation of several downstream intracellular pathways leading to activation of innate and adaptive immune system cells [35-37]. Tolllike receptors control induction of effective adaptive immune responses at several levels, including induction of T-cell priming and DC maturation. Despite their beneficial roles in detecting microbial/viral infections, inappropriate engagement of these receptors on $B$ cells may initiate and/or perpetuate autoimmunity (Fig. 1).

Toll-like receptor activation is required for defense against microbial infections, but on the other hand TLRS are involved in the development of many other noninfectious diseases. Recent reports have indicated that in humans dysfunction in TLR signaling has been associated with several autoimmune diseases by sensing im- mune complexes such as small ribonucleotide proteins composed of self-nucleotides. For example, TLR2 and TLR4 pathways have been implicated in arthritis, type I diabetes mellitus and atherosclerosis [38, 39] whereas TLRs 1-6 are expressed by rheumatoid arthritis (RA) synovial fibroblasts and are thought to provoke joint inflammation in RA [40]. Furthermore, the TLR7 and 9 pathways are associated with both human and mouse models of SLE and systemic sclerosis (SSc) [6, 13, 27, 41]. Moreover, TLR8 mRNA is upregulated in patients with Sjogren's syndrome, pointing to a possible role for TLR8 in the autoimmune processes [21]. Similarly, genetic modifications that lead to a duplication of the TLR7 gene or over-expression of transgenic TLR7 are associated with exacerbated lupus-like symptoms in murine models [16]. However, the mechanisms that normally prevent self-ligands from reaching intracellular TLRs remain poorly defined. It is possible that in autoimmune diseases, self-nucleic acids are protected from degradation by extracellular and endosomal DNA by being part of immune complexes, similarly to how viral genomes are thought to be protected by viral capsids. Alternatively, antibody-mediated uptake might deliver ligands more efficiently to TLRs, by simply concentrating otherwise scarce ligands or by accelerating endosomal maturation such that self-ligands are not degraded before TLR recognition. Moreover, TLRs have been suggested to contribute to the sustained chronic inflammation associated with autoimmune pathogenesis through the induction of antimicrobial genes, inflammatory cytokines and chemokines, upregulation of co-stimulatory molecules and production of specific antibodies against the formerly characterized pathogen proteins [18, 42].

These receptors were initially characterized by their ability to recognize and respond to pathogen-associated molecular patterns that range from bacterial products such as flagellin and lipopolysaccharide (LPS) to nucleic acid structures from viruses and zymosan from fungi. It was later discovered that TLRs also recognize and respond to endogenous ligands that are generated during inflammation from damaged tissue or death cells or actively secreted by activated cells via a non-conventional lysosomal route and lead to the initiation of autoimmune responses and tissue injury [35]. The crucial role of TLRs in host defense is to regulate the innate and adaptive immune responses of epithelial cells at mucosal sites and mediate leukocyte recruitment to infected tissues [43, 44]. Although the TLRs appear to have evolved as a warning system to detect infections, in some cases they can be triggered unexpectedly by self-molecules. This is well established for the intracellular TLRs that detect nucleic acids, including TLR3/7/8/9. These endosomal TLRs act as sensors of foreign nucleic 


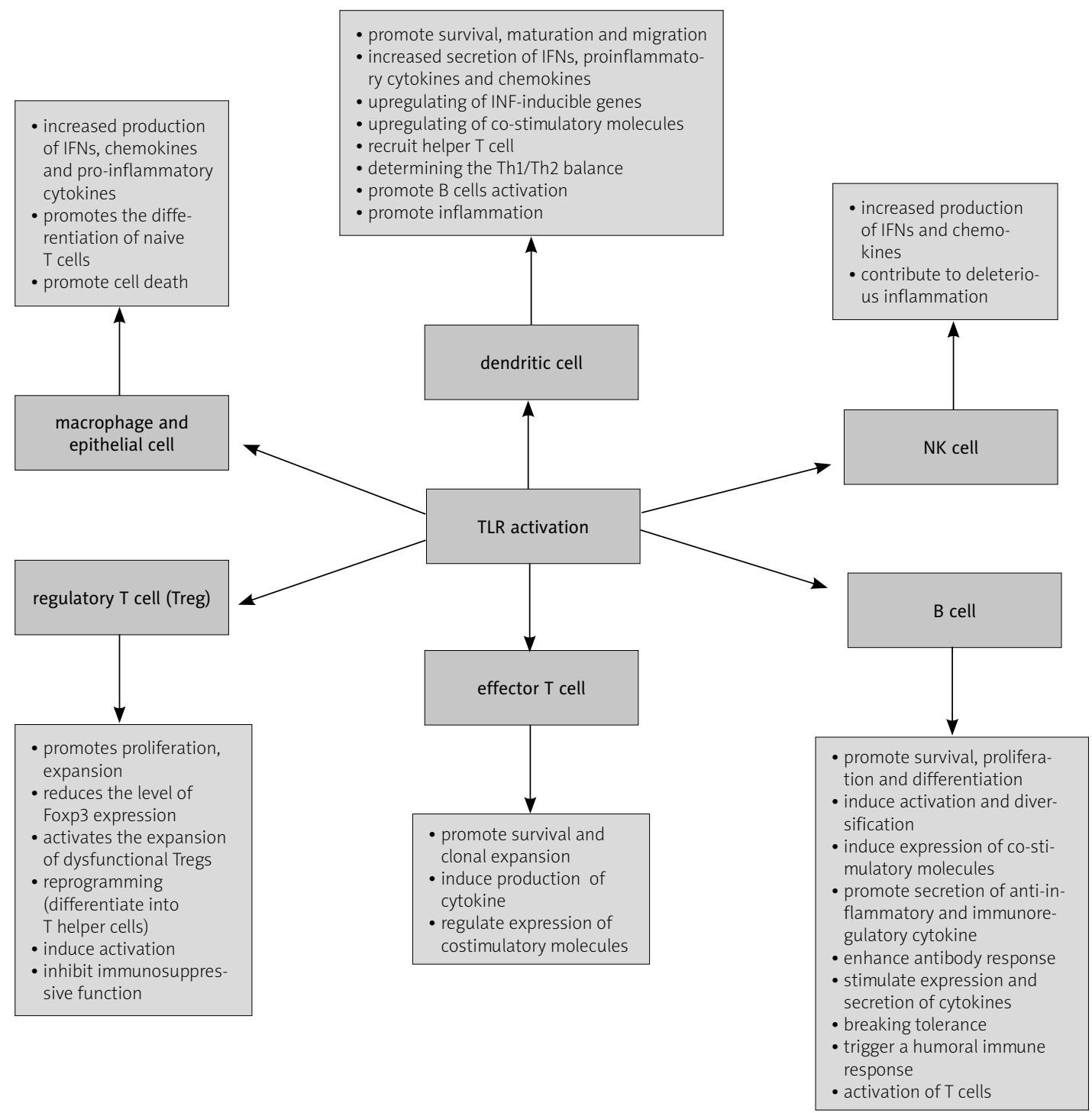

Fig. 1. Role of TLRs in the immune response.

acids and trigger the anti-viral and anti-bacterial (TLR9) immune response by producing type I interferon (IFN) and inflammatory cytokines [19, 22, 27].

The endosomal TLRs are primarily expressed in different DC subsets, where their activation induces type I interferons and leads to upregulation of IFN-inducible genes and secretion of proinflammatory cytokines such as IL-1, IL- 6 and TNF- $\alpha$ [17]. TLR3 might be important for a prolonged response and the initiation of the adaptive immune responses, whereas TLR7 and 9 might trigger a quick IFN response. TLRs regulate gene expression in DCs through a conserved signaling pathway that leads to the activation of several transcription factors, including NF- $\kappa B$, mitogen-activated protein kinases (MAPK), and IFN regulatory factors. Stimulation of TLRs can af- fect both the function and phenotype of DCs, and it is a critical factor leading to conversion of the "immature DC" into "mature DC" cells by upregulation of costimulatory molecules on the surface of DCs [42]. The maturation processes includes expression of the B7 family of molecules and also up-regulation of MHC molecules bearing pathogen-derived peptides [18]. In addition to regulating $D C$ functions, TLR engagement also regulates DC survival and apoptotic death. An initial increase in DC survival immediately after activation is necessary to promote T-cell activation. However, the activated DCs must then have a limited lifespan, to regulate the availability of antigens for $T$ cells, to control the immune response, and to prevent autoimmunity. Studies by Pasare et al. [18] have shown that TLR-induced DC maturation is 
required for activation of CD4+ and CD8+ T cells, which control the adaptive immune system response. Similarly, Moseman et al. [45] demonstrated that human pDCs at specific anatomic sites might also silence T-cell immune responses and through TLR9 induce naive CD4+T cells to differentiate into Treg cells. Thereby they are assumed to prevent excessive damage of surrounding tissue at inflammatory regions on the one hand and perpetuate immunostasis of cell surface regions highly exposed to bacterial products on the other hand. Furthermore, recent data suggest a synergistic effect of dual stimulation of TLRs on DCs, which induces both regulatory and proinflammatory cytokine production of naive $T$ cells. In this regard it has been shown that concomitant activation of TLR4 and TLR7 on DCs induced IL-10 production through the Janus kinase pathway [46]. Moreover, it has been demonstrated that combinatory stimulation of TLR6 and TLR2 enforced induction of regulatory $T$ cells producing IL-10, whereas in the same model the simultaneous stimulation of TLR1 and TLR2 resulted in a proinflammatory immune response [47]. Altogether, it appears that the interaction between certain TLRs on DCs is a critical factor directing DCs into a regulatory or proinflammatory state.

Toll-like receptor members can activate immune responses indirectly by inducing maturation of DCs as well as directly by expression on B and T cells. TLRs can be expressed in $T$ cells and serve as co-stimulatory signals in T-cell activation. TLRs are involved in the control of T cells in two different ways. First, TLRs through induction of professional APCs such as DCs and macrophage DCs induce production of cytokines required for T cell differentiation, activate the upregulation of molecules necessary for T cell clonal expansion, and finally increase the surface displaying complex MHCll-antigen, which can stimulate T cells via TCR. Second, TLRs expressed on $T$ cells have a distinct effect on different $T$ cell subtypes, because stimulation of TLRs on naïve CD4+ T cells is involved in T-cell development, induces effector and regulatory functions of $T$ cells, induces their survival and clonal expansion and can reprogram Treg cells to become helper cells $[42,48]$. Activation of TLRs in APCs would lead to the production of IFN- $\alpha$, proinflammatory cytokines such as TNF- $\alpha$, IL- 1 and IL- 6 , and the cytokines IL-12 and IL-18 that instruct Th1 to differentiate, whereas an increased Th2 response was observed in MyD88 deficient mice with impaired TLR signaling [48]. TLR3 is required to generate IFN- $\gamma$-producing CD8+ T cells, and TLR3 and TLR7 are required for clonal expansion of antigen-specific cells. TLR9 activation by CPG induces potent Th1-type innate and adaptive immune responses with prominent release of IFN- $\gamma, \mathrm{IL}-12$, and IL-18 [27]. TLRs are not only involved in T-cell development but they can also reprogram Treg cells to become helper cells. Treg cells display an increased level of TLR expression compared to conventional CD4+ T cells, suggesting that the expansion and function of Treg cells may be closely influenced by TLR ligands. Treg cells express higher levels of TLR2, TLR4, TLR5, TLR7/8, and TLR10 than effector CD4+ CD25-T cells.

On the other hand, TLR3 is not detectable in naturally occurring Treg cells, while it is expressed by effector CD4+ CD25- T cells [49]. TLR2 stimulation not only abrogates suppressive functions of CD4+ Treg cells but also drives the naïve as well as effector Treg population toward the IL-17-producing Th17 phenotype. In a mouse model of arthritis, mice lacking TLR2 showed enhanced histopathological scores of arthritis by a shift in T cell balance from Th2 and T regulatory cells toward pathogenic Th1 cells. Moreover, TLR2 ligand reduces the level of Foxp3 expression in Treg cells and activates the expansion of dysfunctional Treg cells both in vitro and in vivo $[49,50]$. TLR4, in contrast, contributes to more severe disease by modulating the Th17 cell population and IL-17 production. Furthermore, their participation in Treg cell proliferation and function is still controversial [18, 42, 48]. TLR8 and TLR9 triggering of Treg cells inhibits the immunosuppressive function of these cells but does not induce their proliferation or down-regulation of their Foxp3 expression. The effects or functions of CD4+ T cells are regulated by TLRs, and there are many reports of the direct effects of TLR stimulation in these cells, leading to their proliferation, enhanced survival and induction of B cell responses; however, the molecular pathway involved in skewing of CD4+ T cell function is poorly understood.

Naïve and memory B cells expressed different TLRs to generate an adequate response corresponding to their function, ensuring homeostasis in the immune system. Among the intracellular TLRs, TLR7 and TLR9 play a crucial role in the proliferation and activation of autoreactive $B$ cells and their differentiation into plasma cells secreting antibodies specific to chromatin or chromatin-associated antigens and leading to immune pathology [18, 19, 27]. Moreover, stimulation of B cells via the TLRs pathway causes other important changes such as an increase in cytokine production or up-regulation of molecules essential for antigen presentation to (autoreactive) T cells. TLR7, 8 and 9 directly induced the expression of co-stimulatory molecules including CD80, CD86, CD40, and CD58 on the B cell surface. Moreover, two receptors are modulated by these TLRs: CD23, an important molecule for B cell activation is upregulated, while CD32, resulting in inhibition of B cells, is downregulated [28]. Studies by Bourke et al. [51] demonstrated that expression of TLR9 and TLR10 correlates with B-cell 
differentiation and maturation, and, as a result, stimulation through TLRs may create a link between chronic infection and pathogenesis of noninfectious diseases. In another study it was found that TLR9 stimulation by CpG activates the extracellular signal-regulated kinase (ERK) in B cells, which inhibits secretion of autoantibodies by $B$ cells and induces IL-10 expression, which inhibits the expression of the Th1-like IL-12 and IFNs. Ultimately, a well-regulated immune response not only protects against insults, but also maintains tissue homeostasis $[52,53]$.

\section{Role of endosomal Toll-like receptors in the pathogenesis of mixed connective tissue disease}

Mixed connective tissue disease is a multisystem disease characterized by an autoimmune response to nuclear antigens. Although the pathophysiology of MCTD remains incompletely understood, many studies indicate a major role for innate immunity. TLRs, which play a key role in innate responses to infections, are also involved in acute and chronic inflammatory processes induced by endogenous ligands. Activation of these receptors leads to activation of immune cells, most notably B- and T-cells and DCs, and to the inappropriate production of INF- $\gamma,-\alpha,-\beta$, and many inflammatory cytokines such as IL-1, IL-6, IL-12 and TNF- $\alpha$, which may be involved in the pathogenesis of MCTD [4]. Moreover, TLR stimulation through endogenous ligands is increasingly implicated in autoimmune disease onset and progression. Furthermore, the pathology of MCTD also depends on the production of autoantibodies by autoreactive $B$ cells as well as the expression of large amounts of IFN- $\gamma$ mainly by pDCs. Both these processes are regulated by endosomal TLRs such as TLR3, 7, 8 and 9 [54].

The involvement of TLRs in human diseases is not yet as clear as the data from experimental arthritis models. Recent reports have indicated the role of TLR3 in generation of autoantibodies against U1-RNP in murine models of MCTD. Mice deficient in TLR3 (TLR3-/-) do not develop MCTD-like lung disease when treated with 70K and U1 RNA. However, TLR3-/- mice immunized with 70K and U1 RNA developed anti-dsDNA and an autoimmune syndrome characterized by glomerulonephritis typical of SLE. Moreover, in the absence of mDCs, immunization of TLR3-/- mice with 70K and U1 RNA promotes activation of the PDC population through TLR7 and/or TLR8, leading to the development of SLE. Conversely, the relative paucity of lung disease in SLE compared with MCTD could be explained by the ability of TLR9 (and presumably TLR7 and/or TLR8) but not TLR3 to mediate lung-specific immunosuppressive effects [55]. Further- more, in other mouse models of lupus, genetic deletion of endosomal TLRs significantly reduced disease severity and abolished anti-nuclear antibody titers [13].

Overexpression and dysregulation of endosomal TLRs in DCs, particularly TLR7 and 9, induces higher secretion of interferons (in serum) and upregulation of INF-inducible genes, and the highest serum levels of pro-inflammatory cytokines such as IL-6, IL-12 and TNF- $\alpha$ in MCTD patients compared to healthy subjects $[56,57]$. All these inflammatory mediators contribute to the development and maintenance of autoimmunity in MCTD through the chronic activation of autoreactive Band T-cells. The pathogenic role of IFN- $\gamma$ is mediated in part by its ability to induce immune activation, including a positive feedback loop that induces plasma cell maturation and increases autoantibody formation. Pathology in MCTD could also be caused by the ability of this cytokine to directly induce pathogenic effects at the tissue level $[56,58]$. Thus, identifying the positive and negative regulators of the production and function of IFN- $\gamma$ will contribute to better understanding of how to protect the host from various intracellular pathogen infections while limiting the tissue damage caused by unrestrained inflammatory responses. It is also worth noting that the genes encoding TLR7 and TLR8 are located on chromosome $X$, and women have higher expression of type I interferons in response to TLR7/8 agonists, which may be a major factor responsible for the higher prevalence of MCTD in women [16]. Moreover, TLR-derived signals are able to stimulate angiogenesis and tissue remodeling by induction of vascular endothelial growth factor (VEGF), chemokines and cyclooxygenases (COX) [59]. Vascular endothelial growth factor is an important link between angiogenesis and the inflammatory process. High levels of this factor may be associated with pulmonary arterial hypertension (PAH - one of the main causes of earlier death in MCTD patients) and myositis in MCTD, which suggests that an increased level of VEGF may be a potential marker for patients with increased risk of severe, life-threatening complications.

Participation of endosomal TLRs in anti-U1-RNP autoimmunity is likely explained by the ability of the antigen itself to be stimulatory. Co-administration of U1-RNA with a TLR7 antagonist inhibits induction of DC maturation and B- and T-cell activation, suggesting that TLR7 is directly involved in the U1-RNA adjuvant effect [60].

\section{Summary}

Mixed connective tissue disease is a polygenic disease with a high degree of heterogeneity in clinical manifestations. Recent data suggested that the immune signals may participate in determining the tissue tar- 
gets of immune responses, and hence contribute to the clinical disease pattern in individual patients. Increasing experimental evidence suggests that overexpression and deregulation of TLRs initiate many connective tissue diseases. Therefore, understanding the interactions between the U1-RNP complex and TLRs will provide insights into the way certain patients develop anti-U1-RNP autoimmunity and, more importantly, how to effectively target therapies against this autoimmunity response. Moreover, as endosomal TLRs have been implicated in the development of autoimmune diseases, including MCTD, therapeutic modulation of TLR function and signaling pathways is a valid aim for the development of novel therapies.

\section{The author declares no conflict of interest.}

\section{References}

1. Sharp GC, Irvin WS, Tan EM, et al. Mixed connective tissue disease - an apparently distinct rheumatic disease syndrome associated with a specific antibody to an extractable nuclear antigen (ENA). Am J Med 1972; 52: 148-159.

2. Minkin W, Rabhan N. Mixed connective tissue disease. Arch Dermatol 1976; 112: 1535-1538.

3. Ortega-Hernandez OD, Shoenfeld Y. Mixed connective tissue disease: an overview of clinical manifestations, diagnosis and treatment. Best Pract Res Clin Rheumatol 2012; 26: 61-72.

4. Li J, Wang X, Zhang F, et al. Toll-like receptors as therapeutic targets for autoimmune connective tissue diseases. Pharmacol Ther 2013; 138: 441-451.

5. Chauhan SK, Singh VV, Rai R, et al. Distinct autoantibody profiles in systemic lupus erythematosus patients are selecivelt associated with TLR7 and TLR9 upregulation. J Clin Immunol 2013; 33: 954-964.

6. Celhar T, Magalhaes R, Fairhurst AM. TLR7 and TLR9 in SLE: when sensing self goes wrong. Immunol Res 2012; 53: 58-77.

7. Winfield JB, Koffler D, Kunkel HG. Development of antibodies to ribonucleoprotein following short-term therapy with procainamide. Arthritis Rheum 1975; 18: 531-534.

8. Murakami A, Koijma K, Ohya K, et al. A new conformational epitope generated by the binding of recombinant 70-kd protein and U1 RNA to anti-U1 RNP autoantibodies in sera from patients with mixed connective tissue diseases. Arthritis Rheum 2002; 46: 3273-3282.

9. Aringer M, Steiner G, Smolen JS. Does mixed connective tissue disease exist? Yes. Rheum Dis Clin N Am 2005; 31: 411-420.

10. Greindinger EL, Foecking MF, Ranatunga $S$, et al. Apoptotic U1-70 kd is antigenicall distinct from the intact from of the U1-70-kd molecule. Arthritis Rheum 2002; 46: 1264-1269.

11. Hof D, Cheung K, RAM de Rooij DJ, et al. Antibodies specific for apoptotic U1-70K are superior serological markers for mixed connective tissue disease. Arthritis Res Ther 2005; 7: R302-R309.

12. Osnes LT, Nakken B, Bodolay E, et al. Assessment of intracellular cytokines and regulatory cells in patients with autoim- mune diseases and primary immunodeficiencies - novel tool for diagnostics and patient follow-up. Autoimmun Rev 2013; 12: 967-971.

13. O'Reilly S. Innate immunity is systemic sclerosis pathogenesis. Clin Science 2014; 126: 329-337.

14. Hennessey EJ, Parker AE, O'Neil LAJ. Targeting Toll-like receptors: emerging therapeutics? Nat Rev Drug Discov 2010; 9: 293-307.

15. Römmler F, Hammel M, Waldhuber A, et al. Guanine-modified inhibitory oligonucleotides efficiently impair TLR7- and TLR9-mediated immune responses of human immune cells. PLoS One 2015; 10: e0116703.

16. Brencicova E, Diebold SS. Nucleic acids and endosomal pattern recognition: how to tell friend from foe. Front Cell Infect Microbiol 2013; 3: 37.

17. Trivedi S, Greidimger EL. Endosomal toll-like receptors in autoimmunity: mechanisms for clinical diversity. Therapy 2009; 6: 433-442.

18. Pasare C, Medzhitov R. Toll-like receptors: linking innate and adaptive immunity. Microbes Infect 2004; 6: 1382-1387.

19. Sioud M. Innate sensing of self and non-self RNAs by toll-like receptors. Trends Mol Med 2006; 12: 167-176.

20. Lee CC, Avalos AM, Ploegh HL. Accessory molecules for Tolllike receptors and their function. Nat Rev Immunol 2013; 12: 168-179.

21. Krieg AM, Vollmer J. Toll-like receptors 7, 8, and 9: linking innate immunity to autoimmunity. Immunol Rev 2007; 220: 251-269.

22. Chang ZL. Important aspects of Toll-like receptors, ligands and their signaling pathways. Inflamm Res 2010; 59: 791-808.

23. Kang JY, Lee JO. Structural biology of the toll-like receptor family. Annu Rev Biochem 2011; 80: 917-941.

24. Matsushima N, Tanaka T, Enkhbayar P, et al. Comparative sequence analysis of leucine-rich repeats (LRRs) within vertebrate toll-like receptors. BMC Genomics 2007; 8: 124.

25. Bell JK, Mullen GE, Leifer CA, et al. Leucine-rich repeats and pathogen recognition in toll-like receptors. Trends Immunol 2003; 24: 528-533.

26. Kawai T, Akira S. The role of pattern-recognition receptors in innate immunity: update on toll-like receptors. Nat Immunol 2010; 11: 373-384.

27. Gosu V, Basith S, Kwon OP et al. Therapeutic Applications of Nucleic Acids and Their Analogues in Toll-like Receptor Signaling. Molecules 2012; 17: 13503-13529.

28. Berglová I, Krejsek J, Kolácková M, et al. B cell toll-like receptors with respect to the pathogenesis of Sjögren's syndrome. Acta Medica (Hradec Kralove) 2011; 54: 51-57.

29. Takagi M. Toll-like receptor-a potent driving force behind rheumatoid arthritis. J Clin Exp Hematopathol 2001; 51: 77-92.

30. Zarember KA, Godowski PJ. Tissue expression of human Tolllike receptors and differential regulation of Toll-like receptor mRNAs in leukocytes in response to microbes, their products, and cytokines. J Immunol 2002; 168: 554-561.

31. Babu S, Blauvelt CP, Kumaraswami V, Nutman TB. Cutting edge: diminished T cell TLR expression and function modulates the immune response in human filarial infection. I Immunol 2006; 176: 3885-3889. 
32. Parker LC, Prince LR, Sabroe I. Translational mini-review series on Toll-like receptors: networks regulated by Toll-like receptors mediate innate and adaptive immunity. Clin Exp Immunol 2007; 147: 199-207.

33. Schroder M, Bowie AG. TLR3 in antiviral immunity: key player or bystander? Trends Immunol 2005; 26: 462-468.

34. Carpenter S, O'Neill LA. How important are Toll-like receptors for antimicrobial responses? Cell Microbiol 2007; 9: 1891-901.

35. Yu L, Wang L, Chen S. Endogenous toll-like receptor ligands and their biological significance. J Cell Med 2010; 14: 2592 2603.

36. Medzhitov R, Preston-Hurlburt P, Janeway CA Jr. A human homologue of the Drosophila Toll protein signals activation of adaptive immunity. Nature 1997; 88: 394-397.

37. Medzhitov R, Janeway CA Jr. Self-defense: the fruit fly style. Proc Natl Acad Sci U S A 1998; 95: 429-430.

38. Devaraj S, Dasu MR, Rockwood J, et al. Increased toll-like re ceptor (TLR) 2 and TLR4 expression in monocytes from patients with type 1 diabetes: further evidence of a proinflammatory state. J Clin Endocrinol Metab 2008; 93: 578-583.

39. Kim HS, Han MS, Chuang KW, et al. Toll-like receptor 2 senses beta-cell death and contributes to the initiation of autoimmune diabetes. Immunity 2007; 27: 321-333.

40. Roelofs MF, Wenink MH, Brentano F, et al. Type I interferons might form the link between Toll-like receptor (TLR) 3/7 and TLR4-mediated synovial inflammation in rheumatoid arthritis (RA). Ann Rheum Dis 2009; 68: 1486-1493.

41. Horton CG, Pan ZJ, Farris AD. Targeting toll-like receptors for treatment of SLE. Mediators Inflamm 2010; 2010: pii498890.

42. Abdelsadik A, Trad A. Toll-like receptors on the fork roads between innate and adaptive immunity. Hum Immunol 2011; 72 1188-1193.

43. Picker LJ, Butcher EC. Physiological and molecular mechanisms of lymphocyte homing. Annu Rev Immunol 1992; 10: 561-591.

44. Blander JM, Medzhitov R. Regulation of phagosome maturation by signals from Toll-like receptors. Science 2004; 304 1014-1018.

45. Moseman EA, Liang X, Dawson AJ, et al. Human plasmacytoid dendritic cells activated by CpG oligodeoxynucleotides induce the generation of CD41CD251 regulatory T cells. J Immuno 2004; 173: 4433-4442.

46. Hirata N, Yanagawa Y, Iwabuchi K, et al. Selective regulation of interleukin-10 production via Janus kinase pathway in murine conventional dendritic cells. Cell Immunol 2009; 258: 9-17.

47. Depaolo RW, Tang F, Kim I, et al. Toll-like receptor 6 drives differentiation of tolerogenic dendritic cells and contributes to LcrV-mediated plague pathogenesis. Cell Host Microbe 2008; 4: 350-361.

48. Jin B, Sun T, Yu XH, et al. The effects of TLR activation on T-cell development and differentiation. Clin Dev Immunol 2012 836485 .

49. Kulkarni R1, Behboudi S, Sharif S. Insights into the role of Tolllike receptors in modulation of T cell responses. Cell Tissue Res 2011; 343: 141-152.

50. Xu D, Liu H, Komai-Koma M. Direct and indirect role of Toll-like receptors in T cell mediated immunity. Cell Mol Immunol 2004; 1: 239-246.
51. Bourke E, Bosisio D, Golay J, et al. The toll-like receptor repertoire of human B lymphocytes: inducible and selective expression of TLR9 and TLR10 in normal and transformed cells. Blood 2003; 102: 956-963.

52. Rui L, Vinuesa CG, Blasioli J, Goodnow CC. Resistance to CpG DNA-induced autoimmunity through tolerogenic B cell antigen receptor ERK signaling. Nat Immunol 2003; 4: 594-600.

53. Booth J, Wilson $\mathrm{H}$, Jimbo $\mathrm{S}$, et al. Modulation of B cell responses by Toll-like receptors. Cell Tissue Res 2011; 343: 131-140.

54. Hoffmad RW, Maldonado ME. Immune pathogenesis of mixed connective tissue disease: a short analytical review. Clin Immunol 2008; 128: 8-17.

55. Greidinger EL, Zang Y, Jaimes K, et al. A murine model of mixed connective tissue disease induced with U1 small nuclear RNP autoantigen. Arthritis Rheum 2006; 54: 661-669.

56. Hassan AB, Ronnelid J, Gunnarsson I, et al. Increased serum levels of immunoglobulins, C-reactive protein, type 1 and type 2 cytokines in patients with mixed connective tissue disease. J Autoimmunity 1998; 11: 503-508

57. Bodolay E, Aleksza M, Antal-Szalmas A, et al. Serum cytokine levels and type 1 and type 2 intracellular T cell cytokine profiles in mixed connective tissue disease. J Rheumatol 2002; 29: 2136-2142

58. Szodoray P, Szollosi Z, Gyimesi E, et al. Sarcoidosis in patients with mixed connective tissue disease: clinical, genetic, serological and histological observations. Rheumatol Int 2008; 28 : 743-747.

59. Seledtsov VI, Seledtsova GV. A balance between tissue-destructive and tissue-protective immunities: a role of toll-like receptors in regulation of adaptive immunity. Immunobiology 2012; 217: 430-435.

60. Kattah NH, Kattah MG, Utz PJ. The U1-snRNP complex: structural properties relating to autoimmune pathogenesis in rheumatic diseases. Immunol Rev 2010; 233: 126-145. 